(

Check for

Cite as

Nano-Micro Lett.

(2019) 11:64

Received: 30 May 2019

Accepted: 13 July 2019

Published online: 2 August 2019

(C) The Author(s) 2019

\section{Atomic Layer Deposition-Assisted Construction of Binder-Free Ni@N-Doped Carbon Nanospheres Films as Advanced Host for Sulfur Cathode}

Jun Liu ${ }^{1}$, Aixiang Wei ${ }^{1,2}$, Guoxiang Pan $^{3}$, Qinqin Xiong ${ }^{4}$, Fang Chen ${ }^{5}$, Shenghui Shen ${ }^{6}$, Xinhui Xia ${ }^{6,7 凶}$

$\triangle$ Jun Liu, gdutliu@gdut.edu.cn; Xinhui Xia, helloxxh@zju.edu.cn

1 Guangdong Provincial Key Laboratory of Functional Soft Condensed Matter, School of Materials and Energy, Guangdong University of Technology, Guangzhou 510006, People's Republic of China

2 Department of Information Science, Xinhua College of Sun Yat-sen University, Guangzhou 510520, People's Republic of China

3 Department of Materials Chemistry, Huzhou University, Huzhou 313000, People's Republic of China

4 College of Materials and Environmental Engineering, Hangzhou Dianzi University, Hangzhou 310018, People's Republic of China

5 Department of Chemistry, Zhejiang University, Hangzhou 310027, People's Republic of China

6 State Key Laboratory of Silicon Materials, Key Laboratory of Advanced Materials and Applications for Batteries of Zhejiang Province, and Department of Materials Science and Engineering, Zhejiang University, Hangzhou 310027, People's Republic of China

7 Key Laboratory of Advanced Energy Materials Chemistry (Ministry of Education), College of Chemistry, Nankai University, Tianjin 300071, People's Republic of China

\title{
HIGHLIGHTS
}

- Construct binder-free Ni@N-doped carbon nanospheres (Ni@N-CNSs) films were prepared and used as sulfur host.

- N-doped carbon and nickel layer work together to suppress shuttle of polysulfides.

- Ni@N-CNSs/S electrode shows enhanced rate performance and good cycling life.

ABSTRACT Rational design of hybrid carbon host with high electrical conductivity and strong adsorption toward soluble lithium polysulfides is the main challenge for achieving high-performance lithiumsulfur batteries (LSBs). Herein, novel binder-free Ni@N-doped carbon nanospheres (N-CNSs) films as sulfur host are firstly synthesized via a facile combined hydrothermal-atomic layer deposition method. The cross-linked multilayer N-CNSs films can effectively enhance the electrical conductivity of electrode and provide physical blocking "dams" toward the soluble long-chain polysulfides. Moreover, the doped $\mathrm{N}$ heteroatoms and superficial $\mathrm{NiO}$ layer on $\mathrm{Ni}$ layer can work synergistically to suppress the shuttle of lithium polysulfides by effective chemical interaction/adsorption. In virtue of the unique composite architecture

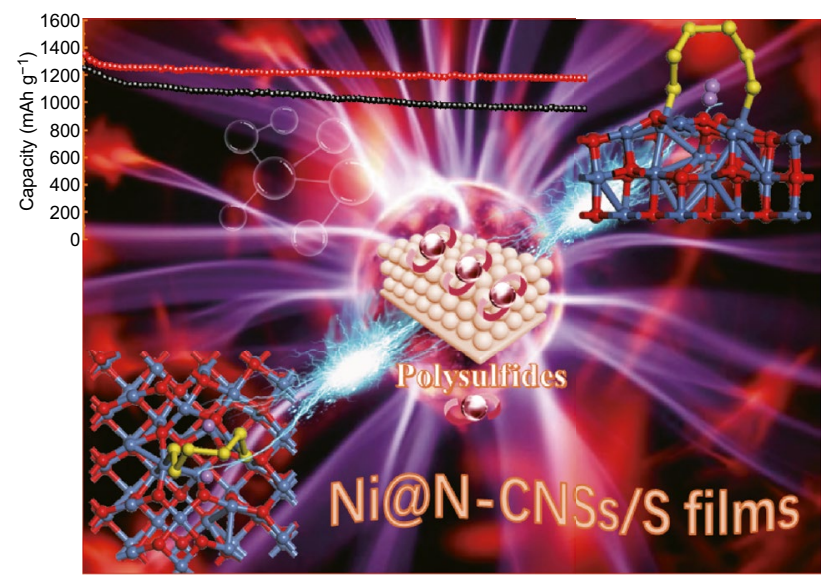
and reinforced dual physical and chemical adsorption to the soluble polysulfides, the obtained $\mathrm{Ni} @ \mathrm{~N}-\mathrm{CNSs} / \mathrm{S}$ electrode is demonstrated with enhanced rate performance $\left(816 \mathrm{mAh} \mathrm{g}^{-1}\right.$ at $\left.2 \mathrm{C}\right)$ and excellent long cycling life $(87 \%$ after 200 cycles at $0.1 \mathrm{C}$ ), much better than N-CNSs/S electrode and other carbon/S counterparts. Our proposed 
design strategy offers a promising prospect for construction of advanced sulfur cathodes for applications in LSBs and other energy storage systems.

KEYWORDS Atomic layer deposition; Nickel; N-doped carbon nanospheres; Sulfur cathode; Lithium-sulfur batteries

\section{Introduction}

Over the past decades, great efforts have been made to develop advanced sulfur cathodes for lithium-sulfur batteries (LSBs) due to its high specific capacity $\left(1675 \mathrm{mAh} \mathrm{g}^{-1}\right)$, large theoretical energy density $\left(2600 \mathrm{Wh} \mathrm{kg}^{-1}\right)$ and low cost [1-4]. Despite promising prospect, the practical application of sulfur cathodes is still blocked by the following problems. (1) High-rate capability of sulfur cathode is not satisfactory on account of low electronic conductivity of active sulfur and final discharge product $\mathrm{Li}_{2} \mathrm{~S}$ [5-7]. Their slow electron transfer not only decreases the reaction efficiency, but also leads to low utilization of active materials. (2) Cycling performance is poor due to the fact that the soluble long-chain lithium polysulfides intermediates show strong "shuttle effect" resulting in fast capacity decay [8]. (3) A volumetric expansion of $\sim 80 \%$ happens to the sulfur cathode during full lithiation to $\mathrm{Li}_{2} \mathrm{~S}$, which is prone to cause structural damage and pulverization of active materials leading to inferior performance [9]. To address these problems, two main strategies including physical block and chemical bonding confinement are used to achieve high performance. One physical strategy is to accommodate sulfur into conductive matrixes/hosts [10-12], which not only act as physical barriers to retard the shuttle of lithium polysulfides, but also offer fast transfer paths for electrons. Additionally, another physical block way is using modified separators [13] or interlayers $[14,15]$ to suppress the shuttle of polysulfides. The latter two ways are usually used as the auxiliary means to restrain the loss of lithium polysulfides. Given all that, sulfur must be combined with advanced conductive hosts, which not only offer large space for accommodation of sulfur, but also possess high electrical conductivity, excellent physical block or chemical adsorption toward soluble lithium polysulfides. Typically, high-performance carbon host is still the first choice for sulfur by virtue of its lightweight, high conductivity, large storage space, cost-effectiveness, and easy modification on structure/composition.

Up to now, lots of carbon hosts (such as carbon nanofibers [16-19], reduced graphene oxides (rGO) [20, 21], carbon nanotubes [18], and carbon nano/micron spheres [22]) have been prepared and combined with sulfur to obtain enhanced performance. It is verified that the conductive carbon host can not only reinforce the electrical conductivity of the whole composite electrode, but also exhibit good suppressing effect toward the soluble lithium polysulfides arising from the physical block/adsorption via micro-, meso- or macropores of carbon [23]. For example, Lu et al. [24] reported 3D micron-porous graphene/sulfur composite cathode with enhanced capacity and good cycling life. In spite of enhanced performance to some extent, the shuttle effect of lithium polysulfides still cannot be completely stopped by the single physical block/adsorption of carbon hosts. In view of this situation, synergistic chemical adsorption strategy needs to be further introduced into carbon/S cathodes by adding polar chemical absorbents. There are two chemical adsorption ways to stabilize the soluble lithium polysulfides. One way is to introduce heteroatoms (e.g., N, P, and $\mathrm{S}$ ) into carbon hosts forming heteroatom-doped carbon hosts $[10,25,26]$. The other way is to rationally combine polar metal $(\mathrm{Ni}, \mathrm{Co})[12]$ or compounds (metal oxides [27, 28], metal sulfides [29, 30], metal nitrides [20], and metal carbides [10], etc.) with carbon hosts forming polar hybrid hosts for sulfur. With the help of first-principle calculations, it has been demonstrated that the heteroatom-doped carbon and polar stoichiometric metal and compounds show higher binding energies $(1.3-3.5 \mathrm{eV})$ with lithium polysulfides than that of the pure carbon hosts $(0.5-1 \mathrm{eV})[27,31]$. It is well accepted that appropriate high binding energy can effectively suppress the shuttle of lithium polysulfides by chemical adsorption, resulting in higher capacity and better cycling life. Therefore, smart integration of heteroatomdoped carbon and polar chemical absorbents into integrated hybrid host is of great importance for achieving high-performance sulfur cathode.

Among the explored carbon hosts, carbon nanospheres (CNSs) have been widely studied as active hosts for sulfur. To date, different carbon nanospheres have been fabricated by different methods including hydrothermal synthesis with sacrificial silica/polystyrene templates, glucose 
decomposition [32, 33], thermal conversion via ZIF-8 template [34], and polyaniline-co-polypyrrole [35], combustion method [36], and sodium dodecyl sulfate-assisted self-assembly method [37]. However, the obtained carbon spheres are powder materials and need combining with additives and polymer binders to form working electrode. This process will increase inner resistance by introducing undesirable interfaces. Therefore, integrated binder-free carbon sphere films become attractive hosts due to binderfree characteristics and multiple blocking "dams" toward lithium polysulfides. To the best of our knowledge, there is no work on the synthesis of integrated N-doped carbon nanosphere (N-CNS) films as host for sulfur. Meanwhile, Ni metal is demonstrated having good chemical adsorption ability to lithium polysulfides. For example, Zhong et al. [12] embedded Ni nanoparticles into puffed rice carbon (PRC) forming PRC/Ni composites host for sulfur with enhanced rate capability. The implantation of Ni not only increases the electrical conductivity, but also synergistically suppresses the shuttling effect of polysulfides. However, their Ni nanoparticles prepared by immersion method show random sizes and cannot be controlled effectively. To overcome this problem, atomic layer deposition (ALD) emerges to produce Ni layer with high reproducibility and uniformity [38], as well as dead-space free. To date, there is no report on the rational combination between N-CNSs and ALD-Ni. Thus, it would be very interesting to explore the integrated Ni@N-CNSs films and their application as host for sulfur cathode.

In this work, we report novel binder-free Ni@N-CNSs films as host for sulfur by a powerful combined hydrothermalALD method. The thin ALD-Ni shell of $\sim 10 \mathrm{~nm}$ is uniformly coated on the N-CNSs skeleton forming advanced host, which is highly compatible with sulfur forming integrated Ni@N-CNSs/S cathode. The Ni@N-CNSs films host not only exhibits high electrical conductivity and large storage room for sulfur, but also possesses synergistic chemical/physical adsorption toward lithium polysulfides. Due to the unique composite architecture, the Ni@N-CNSs/S cathode shows enhanced electrochemical performance with higher capacity, better cycling stability and excellent high-rate capability due to better physisorption and chemisorption abilities and higher conductivity. Our work demonstrates the synergistic effect between N-CNSs and Ni layer toward soluble lithium polysulfides.

\section{Experimental}

\subsection{Preparation of N-Doped Carbon Nanospheres Films}

The N-CNSs films were prepared by a modified hydrothermal method. The nickel foil coated with $\mathrm{ZnO}$ layer $(\sim 10 \mathrm{~nm})$ was used as the substrate. The $\mathrm{ZnO}$ layer was prepared by atomic layer deposition (ALD, Picosun Oy) with Diethyl zinc (DEZ, 99.99\%, Sigma-Aldrich) and $\mathrm{H}_{2} \mathrm{O}$ as the $\mathrm{Zn}$ and $\mathrm{O}$ precursors, respectively. Then, the above substrate was transferred into Teflon-lined autoclave liners, which contained hydrothermal reaction solution consisting of $0.25 \mathrm{M}$ glucose and $0.1 \mathrm{M}$ aniline in aqueous solution. The nickel foil substrate with $\mathrm{ZnO}$ layer was fixed and the autoclave was kept at $180{ }^{\circ} \mathrm{C}$ for $8 \mathrm{~h}$. During the hydrothermal process, the sacrificial $\mathrm{ZnO}$ layer acted as an induced layer to make the glucose/aniline decompose and polymerize on the nickel foil to form carbon nanospheres. After rinse, the samples were annealed at $800{ }^{\circ} \mathrm{C}$ for $3 \mathrm{~h}$ in argon atmosphere to form $\mathrm{N}-\mathrm{CNSs}$ films.

\subsection{Preparation of Ni@N-CNSs Composite Films}

The Ni@N-CNSs composite films were prepared using a SUNALE R-200 ALD reactor (Picosun Oy) with $\mathrm{Ni}(\mathrm{Cp})_{2}$ and $\mathrm{O}_{3}$ (Ozone concentration $\sim 10 \%$, generated in a 500 sccm mixture of oxygen (99.99\%) and nitrogen (99.998\%) as sources for $\mathrm{Ni}$ and oxygen, respectively. The $\mathrm{Ni}(\mathrm{Cp})_{2}$ precursor was put in a stainless steel bottle kept at $165^{\circ} \mathrm{C}$, and the reaction chamber with $\mathrm{N}$-CNSs films was kept at $300{ }^{\circ} \mathrm{C}$ and $14 \mathrm{kPa}$ during the reaction. After ALD process, the samples were annealed at $400{ }^{\circ} \mathrm{C}$ for $2 \mathrm{~h}$ in mixture atmosphere $\left(90 \% \mathrm{Ar}+10 \% \mathrm{H}_{2}\right)$ to form $\mathrm{Ni} @ \mathrm{~N}-\mathrm{CNSs}$ composite films.

\subsection{Preparation of Ni@N-CNSs/S Composite Cathode}

The Ni@N-CNSs composite hosts and sulfur were put into the $\mathrm{CO}_{2}$ supercritical fluid infiltration reactor according to the weight ratio of $1.3: 3$. The pressure was $8.5 \mathrm{MPa}$ and the reactor worked at $250 \mathrm{rpm}$ and kept at $32{ }^{\circ} \mathrm{C}$ for $10 \mathrm{~h}$. After release the bumped $\mathrm{CO}_{2}$ gas, the sample was transferred into Teflon-linked steel autoclave and kept at 
$15{ }^{\circ} \mathrm{C}$ for $12 \mathrm{~h}$ to obtain the final Ni@N-CNSs/S cathode. For comparison, the N-CNS/S sample was also prepared as the same condition. The load mass of $\mathrm{S}$ was about $2.5 \mathrm{mg} \mathrm{cm}^{-2}$ in the $\mathrm{N}-\mathrm{CNSs} / \mathrm{S}$ sample.

\subsection{Materials Characterization}

Morphology and phase compositions of all samples were characterized by field emission scanning electron microscope (SEM, Hitachi S-4700), transmission electron microscope (TEM, FEI Tecnai G2 F20 at 200 kV), X-ray diffraction (XRD, Rigaku D/max $2550 \mathrm{PC}(\mathrm{CuK} \alpha)$ ), X-ray photoelectron spectroscopy (XPS, ESCALABMKLL spectrometer) and Raman spectra (Renishaw Raman microscope under $532 \mathrm{~nm}$ laser excitation). The content of sulfur was detected by thermogravimetric (TG) curves utilizing Netzsch STA 449C thermal analyzer.

\subsection{Electrochemical Characterization}

The binder-free Ni@N-CNSs/S electrode was directly used as the cathode. 2025-type coin cells were applied to assemble test cells. $\mathrm{N}-\mathrm{CNSs} / \mathrm{S}$ electrode was prepared as the same procedure above. The electrolyte was $1 \mathrm{M}$ bis(trifluoromethane) sulfonamide lithium salt (LiTFSI) in a mixed solvent of 1,3-dioxolane (DOL) and 1,2-dimethoxyethane (DME) with a volume ratio of 1:1, including $1 \mathrm{wt} \%$ $\mathrm{LiNO}_{3}$ as an electrolyte additive. The added electrolyte for each electrochemical cell was $20 \mu \mathrm{L} \mathrm{mg}^{-1}$. Lithium metal foil was used as the counter and reference electrode, and a polypropylene microporous film (Cellgard 2300) was used as the separator. 2025-type coin cells were assembled in a glovebox filled with Ar. The discharge/charge performances were tested on a Neware battery program-control test system in a potential range between 1.7 and $2.8 \mathrm{~V}$ at $25^{\circ} \mathrm{C}$. Cyclic voltammetry $(\mathrm{CV})$ measurements were performed with a Princeton TMC 1000 electrochemical workstation (Princeton Applied Research, Co., LTD) in the potential range of $1.7-2.8 \mathrm{~V} \mathrm{(vs.} \mathrm{Li} / \mathrm{Li}^{+}$) at a scan rate of $0.1 \mathrm{mV} \mathrm{s}^{-1}$. Electrochemical impedance spectroscopy (EIS) measurements were conducted in the frequency ranges from $100 \mathrm{kHz}$ to $10 \mathrm{mHz}$ by applying an AC signal of $5 \mathrm{mV}$ on the Princeton electrochemical workstation. The specific capacity was calculated based on the mass of sulfur in the electrode.

\subsection{DFT Calculation}

First-principle based density functional theory (DFT) was performed as implemented in the Vienna ab initio simulation package (VASP). The projector augmented wave (PAW) pseudopotentials and exchange correlation energy functional in generalized gradient approximation (GGA) with the Perdew-Burke-Ernzerhof (PBE) formulation was utilized. The kinetic energy cutoff was set as $500 \mathrm{eV}$. The k-point grids for carbon materials and $\mathrm{NiO}$ were $5 \times 4 \times 2$. In this computation, the $\mathrm{NiO}(200)$ surface was modeled by a bottom fixed slab in a $2 \times 2$ supercell. The vacuum width was set to $15 \AA$ for avoiding the interaction between adjacent slices due to the periodic boundary condition. The pure carbon, pyridinic-N were built by a bottom fixed slab in $3 \times 2$ supercell. The convergence tolerance for this atomic relaxation was set to $1.0 \times 10^{-4} \mathrm{eV} /$ atom for total energy and $0.01 \mathrm{eV} \AA^{-1}$ for force on each atom. The adsorption energies of optimized configurations were calculated by the equation of $E=E_{\text {surf-Li2S6 }}-E_{\mathrm{Li} 2 \mathrm{~S} 6}-E_{\text {surf }}$, where $E_{\mathrm{Li} 2 \mathrm{~S} 6}$ was the energy of the free $\mathrm{Li}_{2} \mathrm{~S}_{6}$ molecular, $E_{\text {surf }}$ was the energy of the surface configuration of pure carbon, pyridinic-N, and NiO. $E_{\text {surf-Li2S6 }}$ was the total energy of the configuration with $\mathrm{Li}_{2} \mathrm{~S}_{6}$ molecular on the corresponding surface.

\section{Results and Discussion}

Figure 1a illustrates the simplified synthetic process of Ni@N-CNSs films. Firstly, the N-CNSs films are fabricated via a modified hydrothermal method. As shown in SEM images (Fig. 1b, c), the N-CNSs films are composed of numerous cross-linked nitrogen-doped carbon nanospheres (N-CNSs) with average diameters of $250 \mathrm{~nm}$. And interestingly, the as-synthesized N-CNSs are closely adhered to each other forming a continuous conductive network, which is favorable to accelerate the electron/ion transportation. Adsorption-desorption isothermal analysis shows that the surface area of N-CNSs films is about $206 \mathrm{~m}^{2} \mathrm{~g}^{-1}$ with a high porosity of about $80.5 \%$ (Fig. S1). ALD-synthesized nickel shell $(\sim 10 \mathrm{~nm})$ is homogeneously deposited on the N-CNSs skeleton forming binderfree Ni@N-CNSs composite films (Fig. 1d, e). It is seen that the appearance of $\mathrm{Ni} @ \mathrm{~N}-\mathrm{CNSs}$ becomes rougher. The 

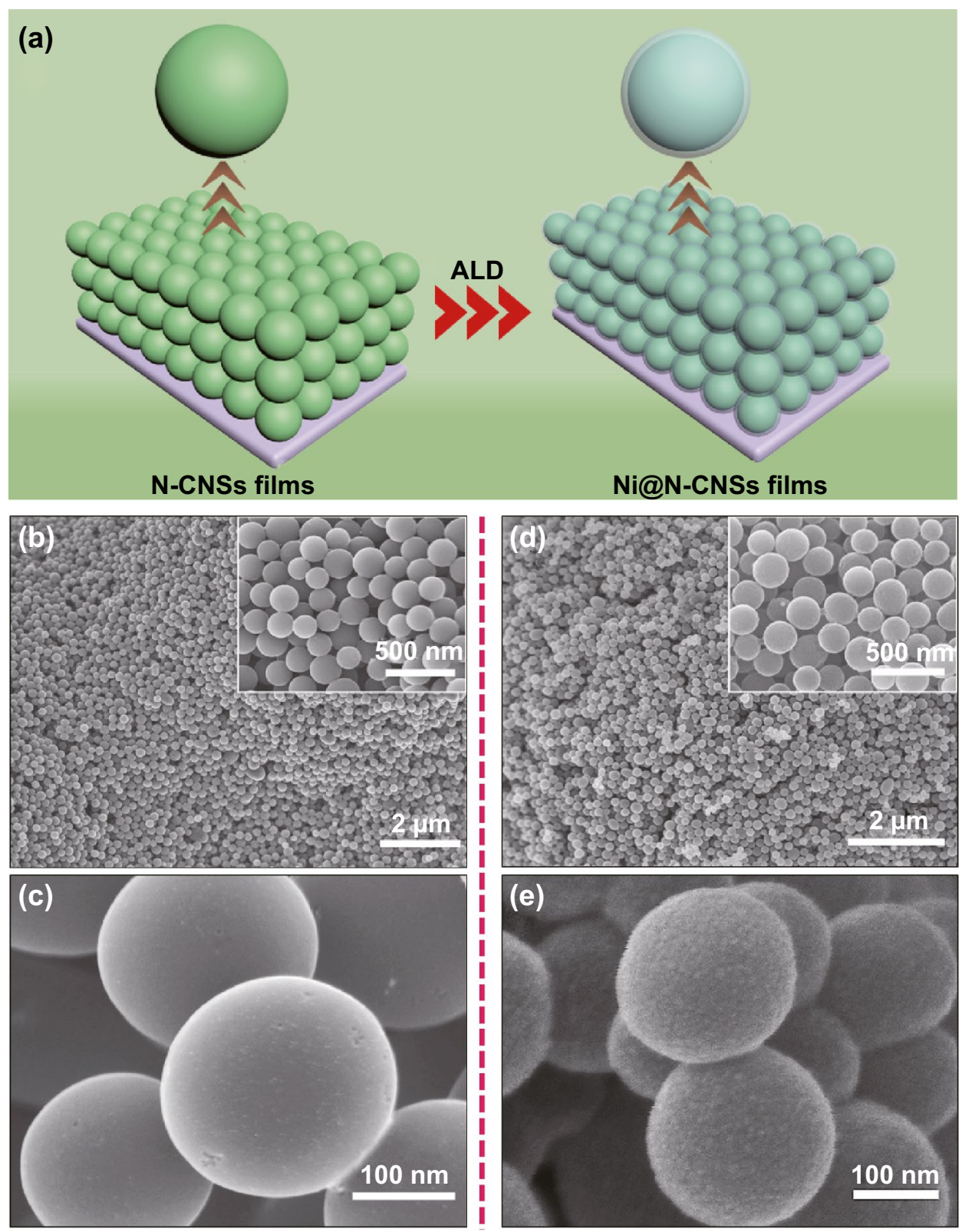

Fig. 1 a Schematic fabrication process of Ni@N-CNSs films. SEM images of b, c N-CNSs and d, e Ni@N-CNSs films

microstructures of samples at different stages are further investigated by TEM and HRTEM tests. The morphology of N-CNSs nanospheres is confirmed as shown in Fig. 2a. Additionally, according to the HRTEM image (Fig. 2b) and SAED pattern (inset in Fig. 2b), there is no obvious crystalline lattice fringe and diffraction rings detected, indicating the amorphous nature of N-CNSs. The doping of nitrogen element is evidenced by EDS elemental mapping images (Fig. 2c). After a facile ALD process, the
$\mathrm{N}-\mathrm{CNSs}$ are homogeneously decorated with Ni layer with a thickness of $\sim 10 \mathrm{~nm}$ (Fig. 2d, e). The measured lattice space of $\mathrm{Ni}$ is $\sim 0.20 \mathrm{~nm}$ (inset in Fig. 2e), corresponding to the (111) crystal plane of cubic Ni phase (JCPDS No. 04-0850). The polycrystalline nature of Ni shell is further verified by characteristic diffraction rings in SAED (inset in Fig. 2d). And the EDS elemental mapping images of $\mathrm{Ni@N-CNSs} \mathrm{films} \mathrm{confirm} \mathrm{that} \mathrm{the} \mathrm{Ni} \mathrm{shell} \mathrm{is} \mathrm{uniformly}$ covered on the surface of N-CNSs (Fig. 2f). 

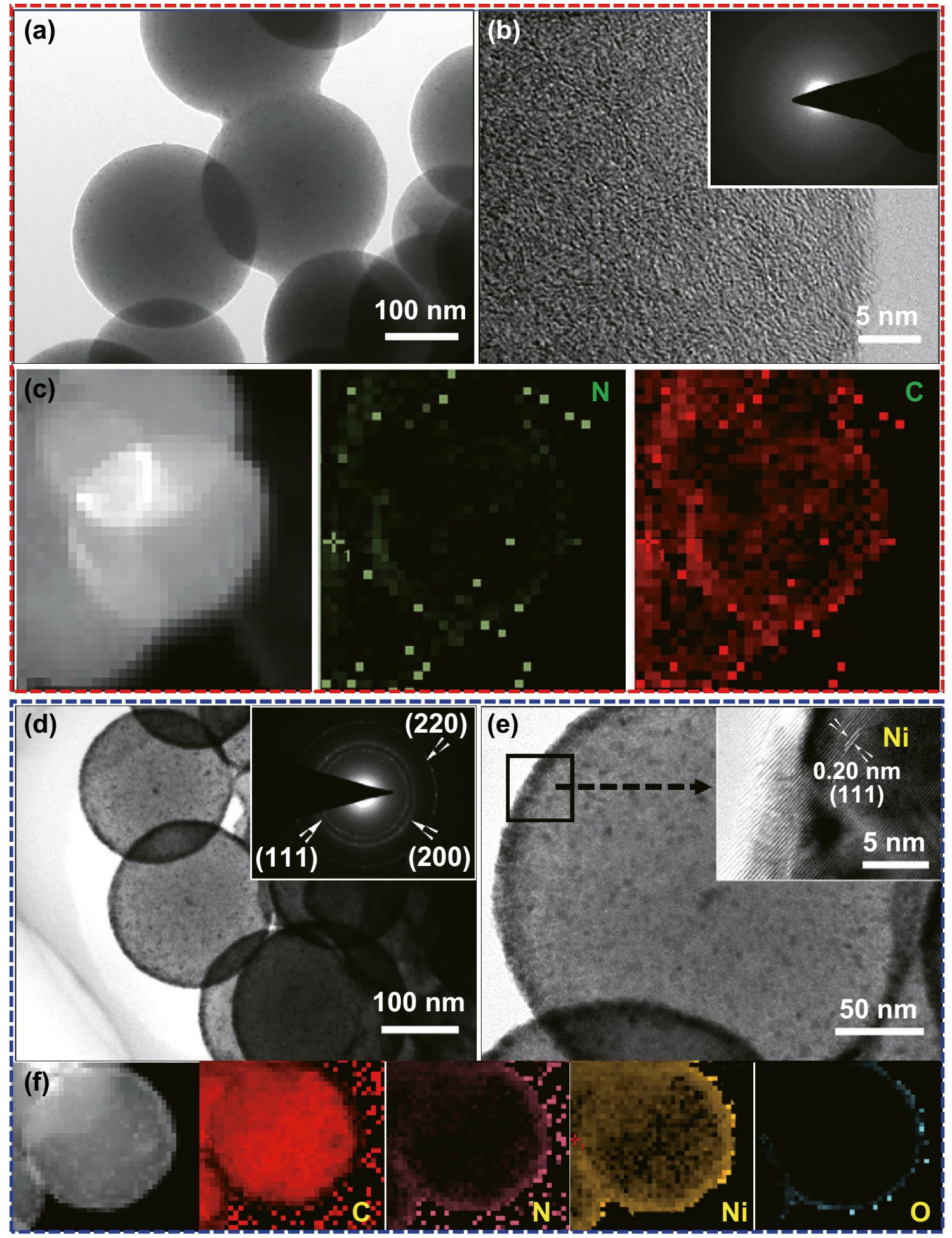

Fig. 2 a, b TEM-HRTEM images and $\mathbf{c}$ EDS elemental mapping images of N-CNSs films. d, e TEM-HRTEM images and f EDS elemental mapping images of $\mathrm{Ni@N-CNSs}$ 

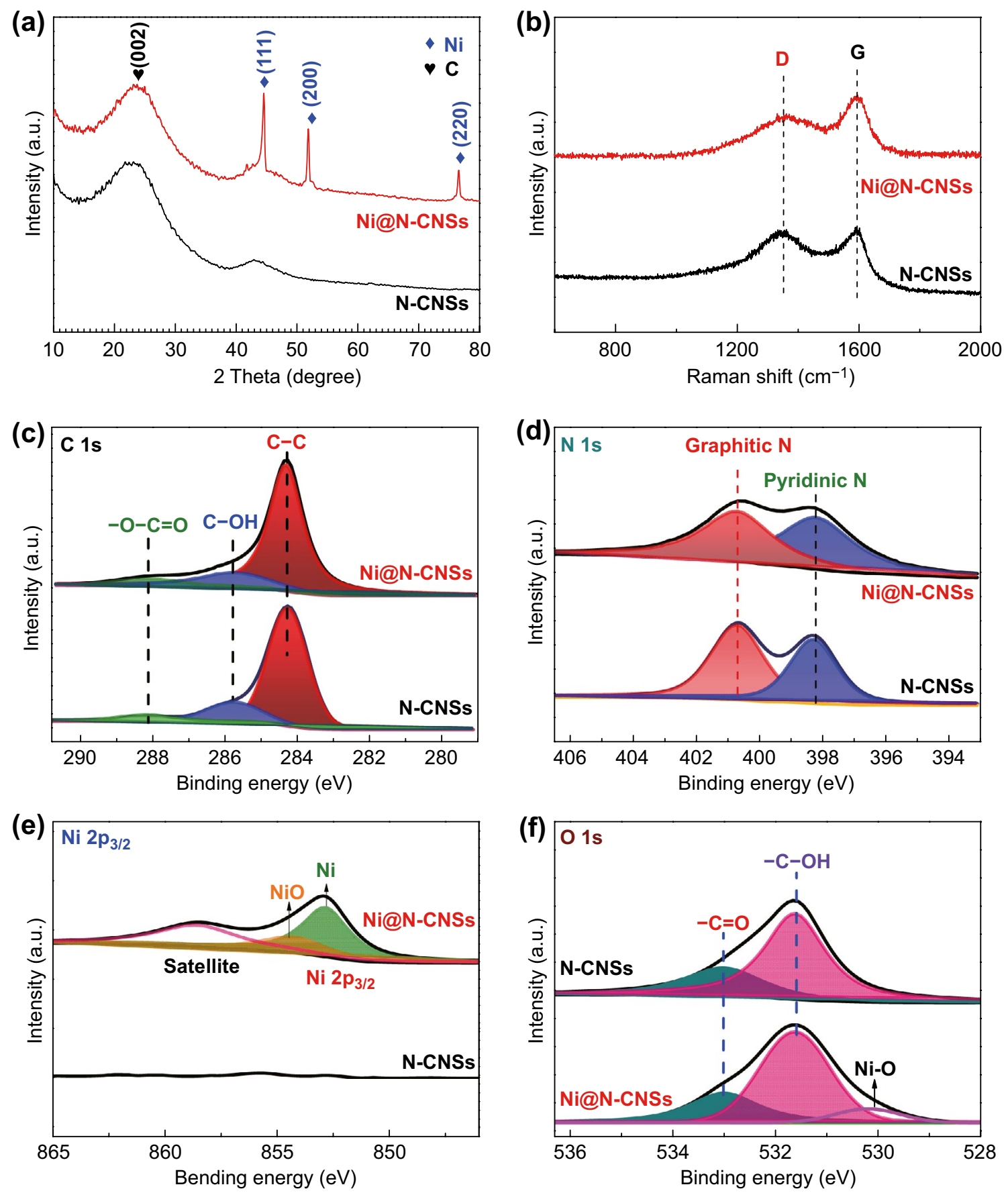

Fig. 3 Phase and composition characterization. a XRD patterns; b Raman spectra; XPS test: c C $1 s$ spectra; d N $1 s$ spectra; e Ni $2 p$ spectra; and f O $1 s$ spectra of N-CNSs and Ni@N-CNSs electrodes

Detailed phase evolution and compositions of N-CNSs and Ni@N-CNSs are detected by XRD, Raman spectra, and XPS spectra (Fig. 3). The XRD pattern of N-CNSs (Fig. 3a) shows two broad diffraction peaks located at $26^{\circ}$ and $43^{\circ}$, corresponding to the (002) and (101) crystal planes of carbon materials (JCPDS No. 75-1621). Apart from these peaks, the Ni@N-CNSs exhibits other three strong characteristic peaks, which can be indexed well with (111), (200), and (220) planes of cubic Ni phase (JCPDS No. 04-0850), confirming the successful deposition of Ni 
shell by ALD method. The above result is also supported by Raman results (Fig. 3b). It is noteworthy that the Ni metal does not show obvious Raman peaks. The Ni@N-CNSs shows larger $I_{\mathrm{G}} / I_{\mathrm{D}}$ ratio than pure N-CNSs, indicating its higher graphitization after the deposition of $\mathrm{Ni}$ shell and annealing process. The surface elemental composition and functional groups of samples are detected by XPS spectra (Fig. 3c-f). Both C $1 s$ spectra (Fig. 3c) contain three characteristic peaks of $-\mathrm{O}-\mathrm{C}=\mathrm{O}(288.1 \mathrm{eV}), \mathrm{C}-\mathrm{OH}(285.8 \mathrm{eV})$, and $\mathrm{C}-\mathrm{C}(284.3 \mathrm{eV})$ bonds. The higher intensity of $\mathrm{C}-\mathrm{C}$ peaks detected in $\mathrm{Ni} @ \mathrm{~N}-\mathrm{CNSs}$ verifies its higher graphitization, consistent with the Raman results above. For the $\mathrm{N} 1 \mathrm{~s}$ spectra (Fig. 3d), two peaks located at 400.7 and $398.3 \mathrm{eV}$ are detected in both samples, corresponding to graphitic $N$ and pyridinic- $N$, respectively, which suggests that the $N$ element is well maintained after the introduction of $\mathrm{Ni}$ shell. As for the Ni $2 p$ spectra (Fig. 3e), it is noteworthy that characteristic peaks of $\mathrm{Ni}(852.9 \mathrm{eV})$ and $\mathrm{NiO}(854.5 \mathrm{eV})$ appear in $\mathrm{Ni} 2 p_{3 / 2}$ spectra, demonstrating the existence of $\mathrm{Ni}$ and $\mathrm{NiO}$ arising from the surface oxidation of $\mathrm{Ni}$ by the air. For the $\mathrm{O} 1 s$ spectra (Fig. $3 \mathrm{f})$, two peaks of $-\mathrm{C}=\mathrm{O}(533.0 \mathrm{eV})$ and $-\mathrm{C}-\mathrm{OH}(531.6 \mathrm{eV})$ are noticed in both $\mathrm{N}-\mathrm{CNSs}$ and

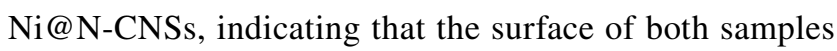
contains $-\mathrm{OH}$ and $-\mathrm{C}=\mathrm{O}$ groups. Meanwhile, a typical peak $(530.1 \mathrm{eV})$ attributed to $\mathrm{Ni}-\mathrm{O}$ bonding is detected in the
$\mathrm{Ni@N-CNSs} \mathrm{due} \mathrm{to} \mathrm{the} \mathrm{superficial} \mathrm{oxidization} \mathrm{layer} \mathrm{of} \mathrm{NiO}$ on the Ni shell. All the results above demonstrate the successful synthesis of the $\mathrm{Ni} @ \mathrm{~N}-\mathrm{CNSs}$ array by a combined hydrothermal-ALD method.

To further explore the electrochemical application of $\mathrm{N}-\mathrm{CNSs}$ and Ni@N-CNSs hosts for LSBs, sulfur is infiltrated into both hosts to obtain N-CNSs/S and Ni@N$\mathrm{CNSs} / \mathrm{S}$ via a supercritical fluid infiltration (SFI) method (Fig. 4a). Notice that sulfur is homogeneously accommodated into the carbon nanospheres and their diameter increases up to $280 \mathrm{~nm}$ with no aggregated sulfur particles (Fig. 4b, c). Similar morphology is observed for the $\mathrm{N}-\mathrm{CNSs} / \mathrm{S}$ sample (Fig. S2). The existence of sulfur in both samples can also be verified by XRD patterns (Fig. 4d). Apart from the peaks of Ni foil substrate and N-CNSs/ $\mathrm{Ni}$ @-CNSs hosts, a series of characteristic peaks of $\mathrm{S}$ are detected in both N-CNSs/S and Ni@N-CNSs/S, supported by XPS characteristic peaks [63.6 eV $\left(2 p_{1 / 2}\right)$ and $164.8 \mathrm{eV}$ $\left.\left(2 p_{2 / 3}\right)\right]$ of sulfur in the $\mathrm{S} 2 p$ spectra (Fig. $\left.4 \mathrm{e}\right)$. Additionally, TEM images of Ni@N-CNSs/S and N-CNSs/S (Figs. 5a, $\mathrm{b}$ and S3a) also demonstrate the good accommodation of sulfur in the Ni@N-CNSs and N-CNSs hosts. For Ni@N$\mathrm{CNSs} / \mathrm{S}$, the Ni shell are homogeneously covered up due to the introduction of sulfur and the surface becomes smoother. EDS elemental mapping images of Ni@N-CNSs/S (Fig. 5c)
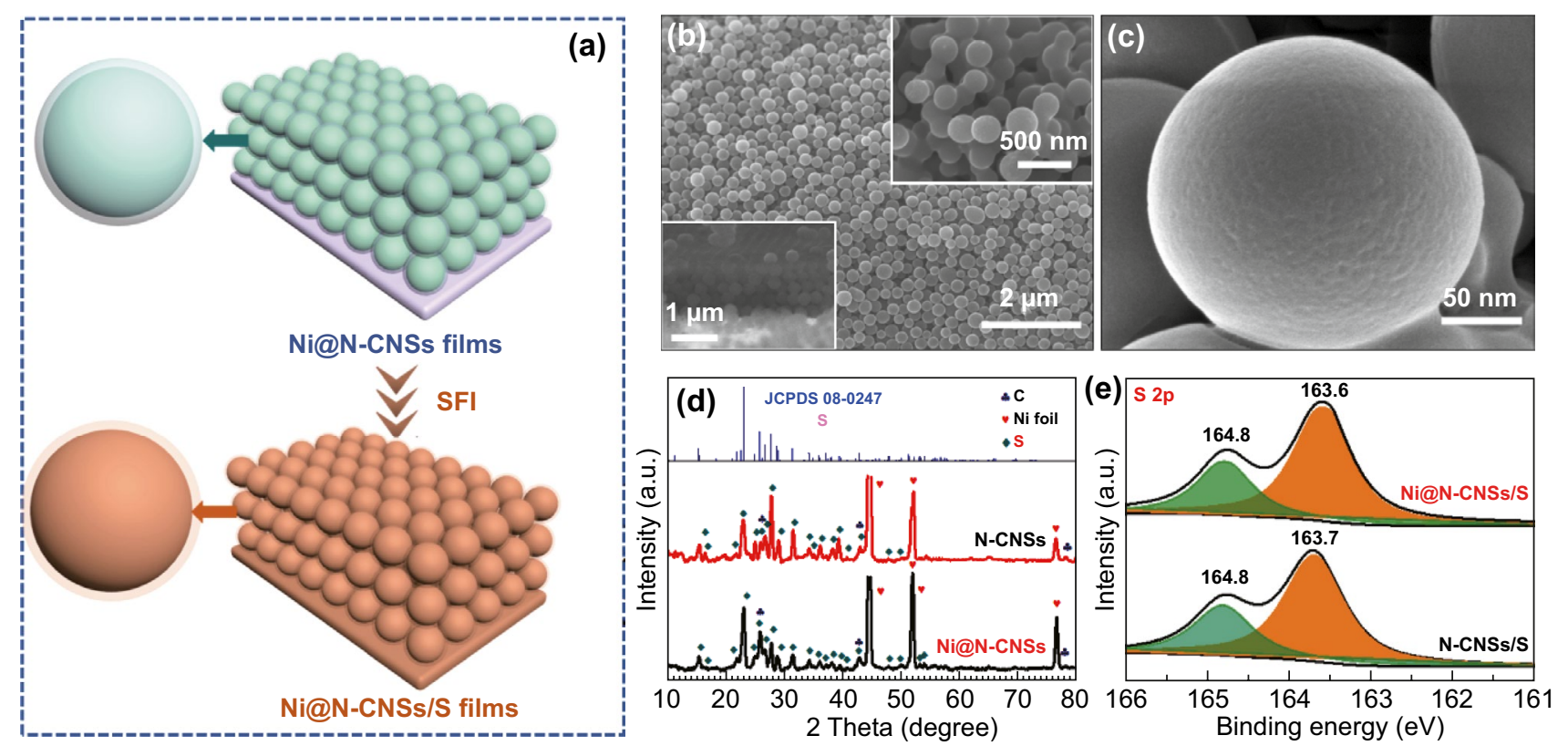

Fig. 4 a Schematic fabrication process of Ni@N-CNSs/S electrode. b, c SEM images of Ni@ N-CNSs/S electrode. d XRD patterns and e S 2 $p$ XPS spectra of N-CNSs/S and Ni@N-CNSs/S electrodes 

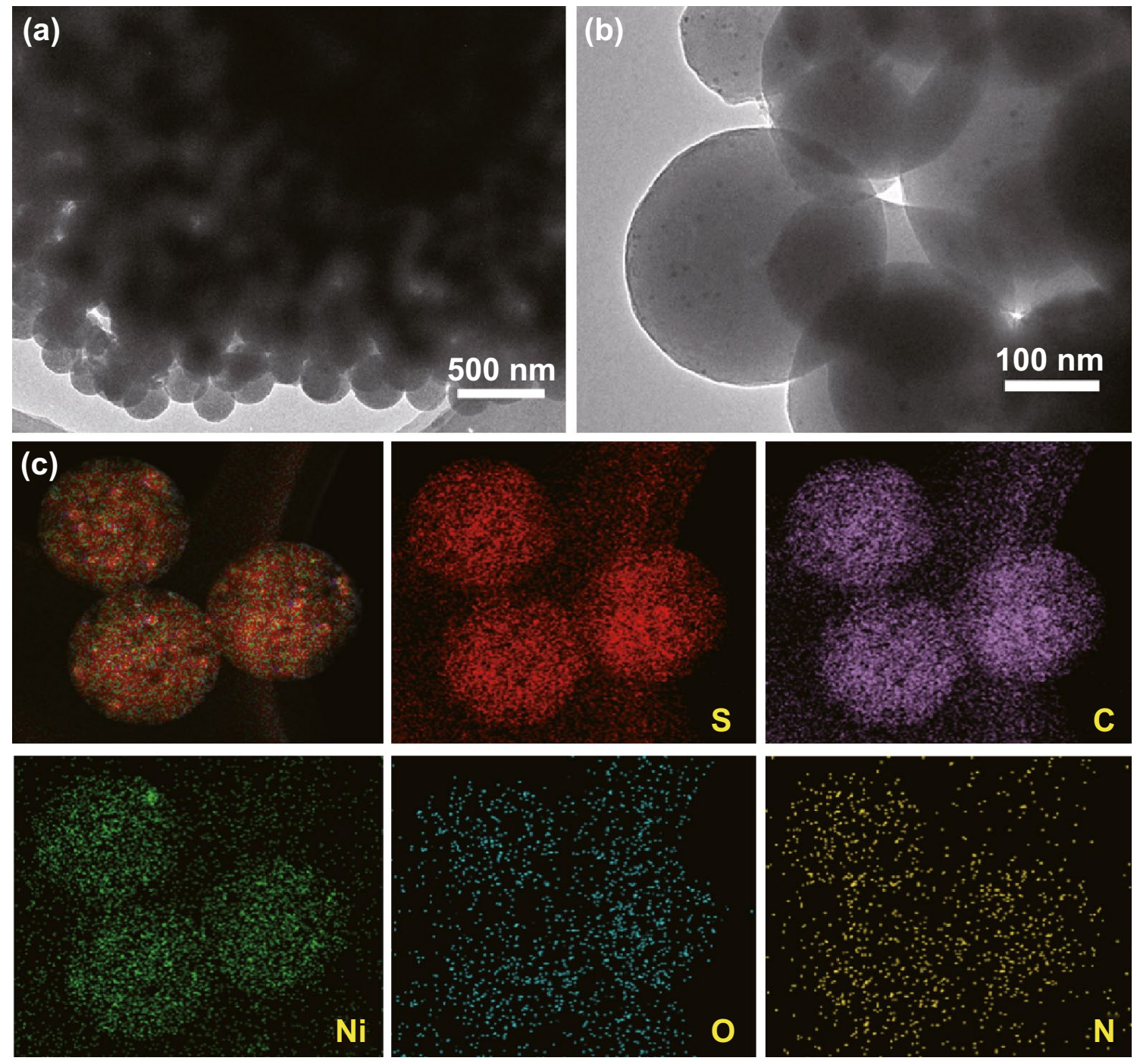

Fig. 5 a, b TEM images and $\mathbf{c}$ EDS elemental mapping images of $\mathrm{Ni} @ \mathrm{~N}-\mathrm{CNSs} / \mathrm{S}$

also confirm the successful preparation of Ni@ N-CNSs host. The element $\mathrm{O}$ is due to the presence of $-\mathrm{OH}$ and $-\mathrm{C}=\mathrm{O}$ groups at the surface $\mathrm{N}-\mathrm{CNSS}$ and superficial $\mathrm{NiO}$ at the surface of Ni layer, which is consistent with the XPS results above. From the TGA results (Fig. S3b), we can calculate that the contents of sulfur in N-CNSs/S and Ni@N-CNSs/S are 66.7 and $68.9 \mathrm{wt} \%$, respectively.

The electrochemical performances of N-CNSs/S and $\mathrm{Ni@N-CNSs/S} \mathrm{electrodes} \mathrm{are} \mathrm{tested} \mathrm{via} \mathrm{cyclic} \mathrm{voltam-}$ metry $(\mathrm{CV})$ and galvanostatic charge/discharge measurements in a voltage range of $1.7-2.8 \mathrm{~V}$ at $25^{\circ} \mathrm{C}$. The overall electrochemical results are shown in Fig. 6. Figure 6a presents the 2nd CV curves of N-CNSs/S and Ni@N-CNSs/S electrodes at a scan rate of $0.1 \mathrm{mV} \mathrm{s}^{-1}$. Both electrodes exhibit two pairs of redox reaction peaks, which correspond to the conversion reactions of sulfur/long-chain polysulfides $\left[\mathrm{S}_{8} / \mathrm{Li}_{2} \mathrm{~S}_{x}(x=4-8)\right]$ and short-chain polysulfides/ $\mathrm{Li}_{2} \mathrm{~S}\left[\mathrm{Li}_{2} \mathrm{~S}_{x}(x<4) / \mathrm{Li}_{2} \mathrm{~S}\right]$. Obviously, the Ni@N-CNSs/S electrode exhibits higher peak density and smaller voltage separation with narrower polarization, indicating its higher reversibility and faster reaction kinetics during the charging/discharging processes. From EIS analysis (Fig. 6b), the charge transfer resistance of $\mathrm{Ni@N-CNSs/S} \mathrm{electrode} \mathrm{is}$ approximately $102 \Omega$, much smaller than that of N-CNSs/S electrode $(220 \Omega)$, indicating its accelerated electrochemical reaction kinetics and better rate capability (Fig. 6c-e) 

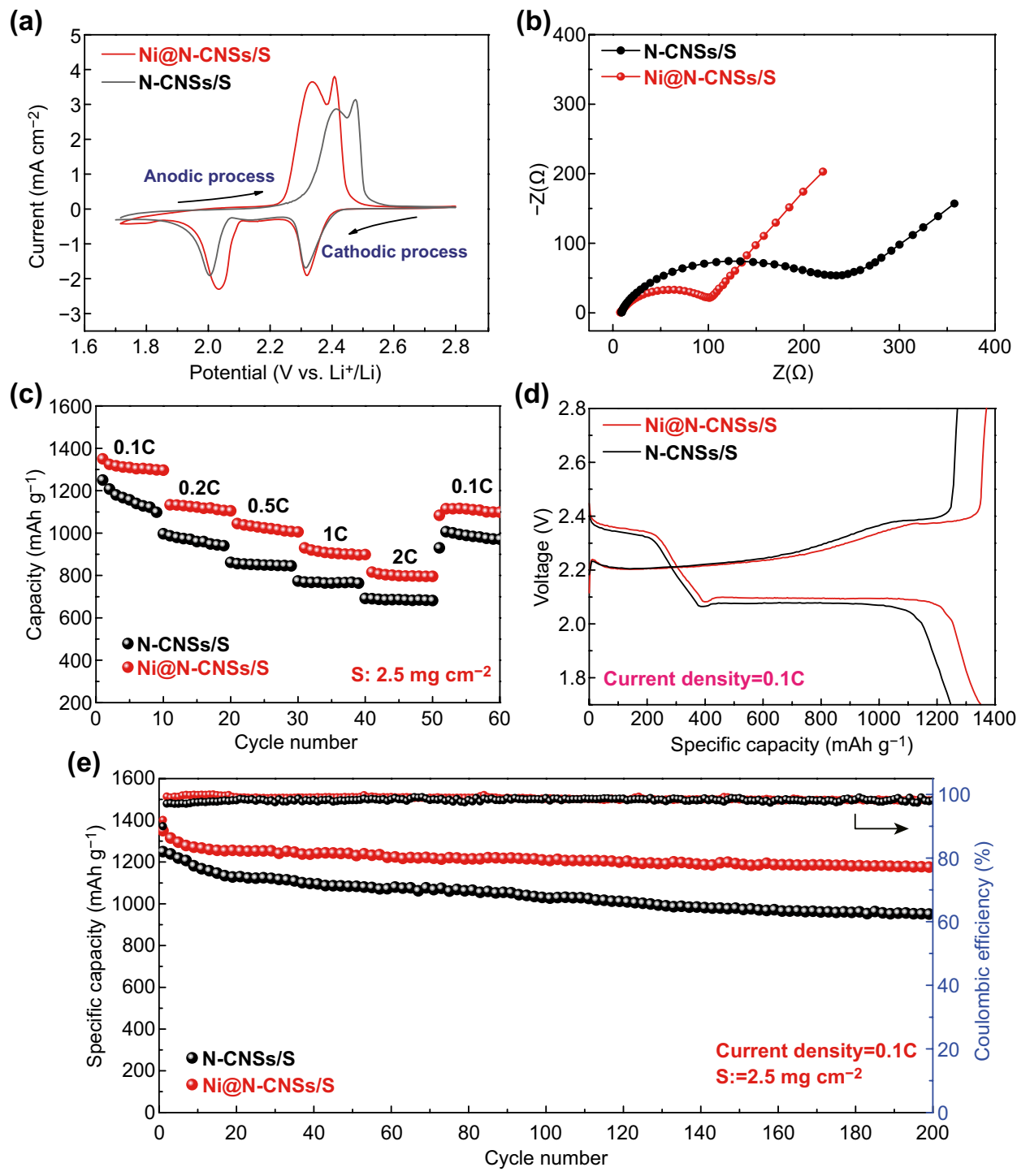

Fig. 6 Electrochemical characterization of $\mathrm{N}-\mathrm{CNSs} / \mathrm{S}$ and $\mathrm{Ni} @ \mathrm{~N}-\mathrm{CNSs} / \mathrm{S}$ electrodes. a CV curves at a scan rate of $0.1 \mathrm{mV} \mathrm{s}{ }^{-1}$ at the second cycle. b Nyquist plots. c Rate capability with a sulfur loading of $2.5 \mathrm{mg} \mathrm{cm}^{-2}$. d Discharge/charge profiles at $0.1 \mathrm{C}$. e Cycling performance and coulombic efficiency at $0.1 \mathrm{C}$

due to the cooperative work of the nitrogen active sites and conductive Ni network. The rate performance of both electrodes ( $\mathrm{S}$ mass loading: $2.5 \mathrm{mg} \mathrm{cm}^{-2}$ ) at different current rates from 0.1 to $2 \mathrm{C}$ is illustrated in Fig. 6c. The Ni@N$\mathrm{CNSs} / \mathrm{S}$ electrode delivers higher discharge capacities (1350, $1133,1045,930$, and $816 \mathrm{mAh} \mathrm{g}^{-1}$ at $0.1,0.2,0.5,1$, and $2 \mathrm{C})$ than the N-CNSs/S counterpart $(1250,987,856,769$, and $690 \mathrm{mAh} \mathrm{g}^{-1}$ at $0.1,0.2,0.5,1$, and $2 \mathrm{C}$ ). Obviously, the Ni@N-CNSs/S electrode exhibits obvious high-rate improvement, owing to its better conductive network and enhanced physisorption and chemisorption ability to the soluble polysulfide species. Both discharging curves of N-CNSs/S and Ni@N-CNSs/S electrodes (0.1 C) show two charge/discharge plateaus (Fig. 6d), which is consistent with the $\mathrm{CV}$ results above. The Ni@N-CNSs/S electrode shows higher discharge plateau voltage and lower charge plateau voltage, as compared with $\mathrm{N}-\mathrm{CNSs} / \mathrm{S}$ electrode, implying its smaller voltage drop and lower polarization. Moreover, the Ni@N-CNSs/S electrode shows superior cycling stability with an initial discharging capacity of $1350 \mathrm{mAh} \mathrm{g}^{-1}$ with a capacity retention of $\sim 87 \%$ at $0.1 \mathrm{C}$ after 200 cycles (Fig. 6e), while the corresponding value of the $\mathrm{N}-\mathrm{CNSs} / \mathrm{S}$ 
electrode is $1250 \mathrm{mAh} \mathrm{g}^{-1}$ with a capacity retention of $76 \%$. The obtained values of $\mathrm{Ni} @ \mathrm{~N}-\mathrm{CNSs} / \mathrm{S}$ electrode are also much better than that of other carbon/S powder electrodes (e.g., hollow carbon nanospheres/S [37], carbon spheres/S [39], S/C nanospheres [36, 40]) (Table S1). As for the Coulombic Efficiency (CE) analysis, after 200 cycles, the CE value of $\mathrm{Ni@N-CNSs/S} \mathrm{electrode} \mathrm{maintains} \mathrm{98.5 \% ,} \mathrm{higher}$ than that of N-CNSs/S (97.8\%). After 200 cycles at $0.1 \mathrm{C}$, the whole composite structure is basically well preserved (Fig. S4). In addition, after 500 cycles at $1 \mathrm{C}$, the Ni@NCNSs/S electrode shows a capacity of $\sim 699 \mathrm{mAh} \mathrm{g}^{-1}$, higher than that of the N-CNSs/S counterpart (477 $\mathrm{mAh} \mathrm{g}^{-1}$ ), indicating its good high-rate stability (Fig. S5). When the loading mass of sulfur is increased up to $4.5 \mathrm{mg} \mathrm{cm}^{-2}$, the $\mathrm{Ni@N-CNSs/S} \mathrm{electrode} \mathrm{still} \mathrm{exhibits} \mathrm{good} \mathrm{cycling} \mathrm{life}$ with a capacity of $839 \mathrm{mAh} \mathrm{g}^{-1}$ at $0.1 \mathrm{C}$ after 500 cycles (Fig. S6), better than that of the N-CNSs/S counterpart (640 $\mathrm{mAh} \mathrm{g}^{-1}$ at $0.1 \mathrm{C}$ after 500 cycles).

The enhanced performance is also verified by morphology comparison of lithium metal anode after cycling at $0.1 \mathrm{C}$ for 100 cycles. As shown in Fig. S7a, c, the Li anode cycled with Ni@N-CNSs/S cathode for 100 cycles shows lower roughness than the counterpart coupled with $\mathrm{N}-\mathrm{CNSs} / \mathrm{S}$ cathode, verifying the effective suppression of the polysulfides due to the synergistic effect between $\mathrm{N}$-doped CNSs architecture and polar superficial NiO layer on Ni. By comparing the color of separators after cycles, the cycled separator with $\mathrm{Ni@N-CNSs/S} \mathrm{cathode} \mathrm{exhibits} \mathrm{a} \mathrm{color} \mathrm{of} \mathrm{lighter}$ yellow (inset in Fig. S7a, c). Furthermore, according to the EDS mapping images of S (Fig. S7b, d), less polysulfides have diffused to the Li anode coupled with $\mathrm{Ni@N-CNSs/S}$ cathode, indicating its better suppressing effect on the soluble polysulfides.

The outstanding electrochemical performance of $\mathrm{Ni@N-}$ $\mathrm{CNSs} / \mathrm{S}$ electrode is mainly owing to the following positive factors: (1) Binder-free conductive characteristics. The continuous conductive network of Ni@N-CNSs without additives and binders can effectively decrease inner charge transfer resistance by avoiding undesirable interfaces, and thereby enhance the electrical conductivity of the electrode and reaction kinetics [41-43]. (2) Physical adsorption and block to the soluble lithium polysulfides. Cross-linked multilayer carbon nanospheres films provide a physical "dam" to entrap the soluble polysulfide species and decreases the irreversible capacity during the charge/discharge processes. (3) Chemical adsorption to the polysulfides. Doped nitrogen heteroatoms and polar superficial $\mathrm{NiO}$ on $\mathrm{Ni}$ cannot only enhance the electronic conductivity of the matrix, but also synergistically exhibit strong chemical interaction with the long-chain polysulfides, resulting in higher utilization of active materials and stable cycling life during the redox reaction processes. In our case, the function of $\mathrm{Ni}$ shell should be highlighted as follows. On one hand, the Ni layer on N-CNSs can establish good electron transfer path from the bottom to the top forming omnibearing conductive network. According to four-point probe method, the electrical conductivity of Ni@N-CNSs is $8.7 \times 10^{4} \mathrm{~S} \mathrm{~m}^{-1}$, much higher than that of N-CNSs films $\left(0.9 \times 10^{3} \mathrm{~S} \mathrm{~m}^{-1}\right)$. On the other hand, the superficial $\mathrm{NiO}$ at the $\mathrm{Ni}$ layer has strong adsorption ability to the soluble polysulfides to maintain good electrochemical performance. Figure 7 shows the adsorption energetics of the optimized adsorption configurations with $\mathrm{Li}_{2} \mathrm{~S}_{6}$ molecular on thin $\mathrm{NiO}$ layer ( $\mathrm{NiO}$ is the oxidized layer on the surface of $\mathrm{Ni}$ ), pure carbon and $\mathrm{N}$-doped carbon. The adsorption energy of $\mathrm{Li}_{2} \mathrm{~S}_{6}$ on $\mathrm{NiO}$ is about $-2.86 \mathrm{eV}$, higher than the values obtained from $\mathrm{N}$-doped carbon $(-0.98 \mathrm{eV})$ and pure carbon $(-0.55 \mathrm{eV})$ as well as the adsorption energy value between 1,2-dimethoxyethane (DME) $-\mathrm{Li}_{2} \mathrm{~S}_{6}(-0.77 \mathrm{eV}$ ) [44]. It suggests that the presence of $\mathrm{N}$ doping and superficial $\mathrm{NiO}$ on the $\mathrm{Ni}$ layer coating can greatly enhance the chemical affinity to the soluble $\mathrm{Li}_{2} \mathrm{~S}_{6}$, leading to boosted rate performance and long-term cycles.

High-resolution $\mathrm{S} 2 p$ spectra of pristine $\mathrm{Li}_{2} \mathrm{~S}_{6}$ and $\mathrm{Ni} @ \mathrm{~N}$ CNSs- $\mathrm{Li}_{2} \mathrm{~S}_{6}$ are shown in Fig. S8. The pristine $\mathrm{Li}_{2} \mathrm{~S}_{6}$ exhibits
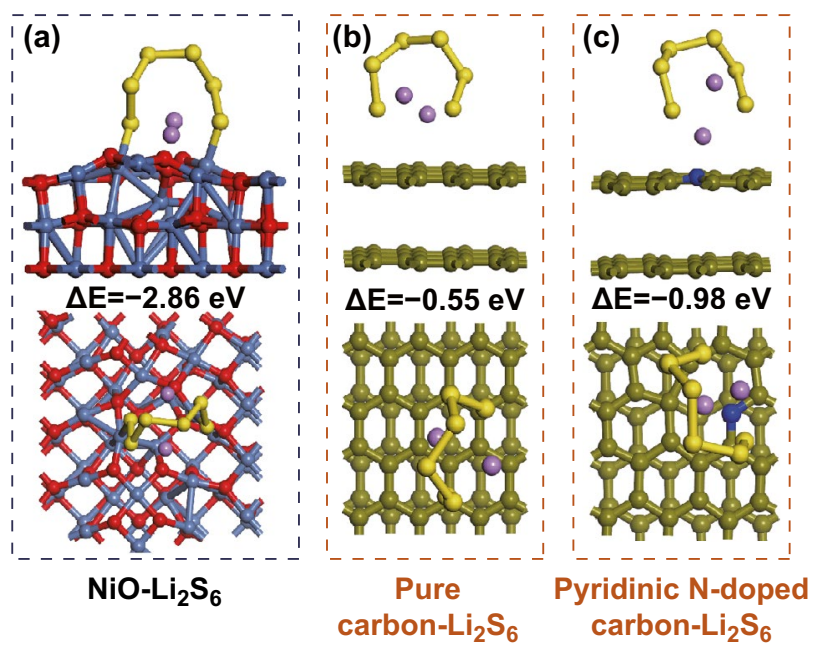

Fig. 7 DFT calculations on adsorption energies between $\mathrm{NiO}$, pure carbon, and pyridinic-N-doped carbon with $\mathrm{Li}_{2} \mathrm{~S}_{6}$ 
two typical sulfur environments at 161.6 and $163.1 \mathrm{eV}$ owing to terminal $\left(\mathrm{S}_{\mathrm{T}}^{-1}\right)$ and bridging $\left(\mathrm{S}_{\mathrm{B}}^{0}\right)$ sulfur atoms [45], respectively. In contrast, after coupling with Ni@N-CNSs host, the peak of $\mathrm{S}_{\mathrm{T}}^{-1}$ positively shifts to higher binding energy of $162.9 \mathrm{eV}$, suggesting a decrease in electron density on $\mathrm{S}_{\mathrm{T}}^{-1}$ in $\mathrm{Ni} @ \mathrm{~N}-\mathrm{CNSs}-\mathrm{Li}_{2} \mathrm{~S}_{6}$. This result indicates that $\mathrm{Ni} @ \mathrm{~N}-\mathrm{CNSs}$ host has strong chemical adsorption to $\mathrm{S}_{\mathrm{T}}^{-1}$. An additional $\mathrm{S}_{\mathrm{B}}^{0}-\mathrm{C}$ species is detected at $164.4 \mathrm{eV}$, due to the disproportionation of $\mathrm{Li}_{2} \mathrm{~S}_{6}$ into $\mathrm{S}^{0}$. Furthermore, the peaks $(168.2 \mathrm{eV})$ of polythionate and $\mathrm{S}$-complex species appear in $\mathrm{Ni@N-}$ $\mathrm{CNSs}-\mathrm{Li}_{2} \mathrm{~S}_{6}$ due to intermediate redox reactions [45]. Given all that, it is justified that $\mathrm{Ni} @ \mathrm{~N}-\mathrm{CNSs}$ host can effectively entrap polysulfides to maintain good electrochemical performance. A simple adsorption test was conducted for $\mathrm{Ni} @ / \mathrm{N}$ CNSs and N-CNSs (Fig. S9). After $18 \mathrm{~h}$, the polysulfide solution with Ni@N-CNSs becomes transparent, while the counterpart still shows light yellow. It is indicated that Ni@/ $\mathrm{N}$-CNSs host exhibits much better adsorption ability to the soluble polysulfides.

\section{Conclusion}

In summary, we have proposed a new hydrothermal-atomic layer deposition method to synthesize binder-free Ni@NCNSs films as sulfur host for lithium-sulfur batteries. The binder-free multilayer N-CNSs films not only perform enhanced electronic conductivity, but also provide physical block toward the soluble long-chain polysulfides. With the deposition of ALD-Ni shell, the synergistic work of nitrogen active sites and polar $\mathrm{Ni}$ adsorbents further increases the intrinsic reactivity kinetics during the redox reactions and offers a strong chemical adsorption to the polysulfide intermediates. After rational combination with sulfur, the assynthesized Ni@N-CNSs/S cathode shows enhanced overall electrochemical performance with higher high-rate capacity and better cycling life due to the well-designed hybrid host. Our rational design of binder-free hybrid sulfur host may break the new ground in terms of high-performance cathode for lithium-sulfur batteries.

Acknowledgements This work is supported by National Natural Science Foundation of China (Nos. 51772272 and 51728204), Fundamental Research Funds for the Central Universities (No. 2018QNA4011), Science and Technology Program of Guangdong Province of China (No. 2016A010104020), Pearl River S\&T Nova Program of Guangzhou (No. 201610010116), Qianjiang Talents
Plan D (QJD1602029) and Startup Foundation for Hundred-Talent Program of Zhejiang University.

Open Access This article is distributed under the terms of the Creative Commons Attribution 4.0 International License (http:// creativecommons.org/licenses/by/4.0/), which permits unrestricted use, distribution, and reproduction in any medium, provided you give appropriate credit to the original author(s) and the source, provide a link to the Creative Commons license, and indicate if changes were made.

Electronic supplementary material The online version of this article (https://doi.org/10.1007/s40820-019-0295-8) contains supplementary material, which is available to authorized users.

\section{References}

1. L. Hencz, H. Chen, H.Y. Ling, Y. Wang, C. Lai, H. Zhao, S. Zhang, Housing sulfur in polymer composite frameworks for Li-S batteries. Nano-Micro Lett. 11, 17 (2019). https://doi. org/10.1007/s40820-019-0249-1

2. Y. Zhong, X. Xia, S. Deng, J. Zhan, R. Fang et al., Popcorn inspired porous macrocellular carbon: rapid puffing fabrication from rice and its applications in lithium-sulfur batteries. Adv. Energy Mater. 8, 1701110 (2018). https://doi.org/10.1002/ aenm.201701110

3. Y. Zhong, D. Chao, S. Deng, J. Zhan, R.Y. Fang et al., Confining sulfur in integrated composite scaffold with highly porous carbon fibers/vanadium nitride arrays for high-performance lithium-sulfur batteries. Adv. Funct. Mater. 28, 1706391 (2018). https://doi.org/10.1002/adfm.201706391

4. Y. Zhang, X. Xia, B. Liu, S. Deng, D. Xie et al., Multiscale graphene-based materials for applications in sodium ion batteries. Adv. Energy Mater. 9, 1803342 (2019). https://doi. org/10.1002/aenm.201803342

5. Y. Zhao, Y. Ye, F. Wu, Y. Li, L. Li, R. Chen, Anode interface engineering and architecture design for high-performance lithium-sulfur batteries. Adv. Mater. 31(12), 1806532 (2019). https://doi.org/10.1002/adma.201806532

6. H. Yuan, J.-Q. Huang, H.-J. Peng, M.-M. Titirici, R. Xiang, R. Chen, Q. Liu, Q. Zhang, A review of functional binders in lithium-sulfur batteries. Adv. Energy Mater. 8(31), 1802107 (2018). https://doi.org/10.1002/aenm.201802107

7. S.L. Zhang, B.Y. Guan, H.B. Wu, X.W.D. Lou, Metal-organic framework-assisted synthesis of compact $\mathrm{Fe}_{2} \mathrm{O}_{3}$ nanotubes in $\mathrm{Co}_{3} \mathrm{O}_{4}$ host with enhanced lithium storage properties. NanoMicro Lett. 10(3), 44 (2018). https://doi.org/10.1007/s4082 0-018-0197-1

8. H. Yuan, H.-J. Peng, B.-Q. Li, J. Xie, L. Kong et al., Conductive and catalytic triple-phase interfaces enabling uniform nucleation in high-rate lithium-sulfur batteries. Adv. Energy Mater. 9(1), 1802768 (2019). https://doi.org/10.1002/ aenm.201802768 
9. X. Yu, A. Manthiram, Enhanced interfacial stability of hybridelectrolyte lithium-sulfur batteries with a layer of multifunctional polymer with intrinsic nanoporosity. Adv. Funct. Mater. 29(3), 1805996 (2019). https://doi.org/10.1002/adfm.20180 5996

10. S. Shen, X. Xia, Y. Zhong, S. Deng, D. Xie et al., Implanting niobium carbide into trichoderma spore carbon: a new advanced host for sulfur cathodes. Adv. Mater. 31, 1900009 (2019). https://doi.org/10.1002/adma.201900009

11. W. Li, R. Fang, Y. Xia, W. Zhang, X. Wang, X. Xia, J. Tu, Multiscale porous carbon nanomaterials for applications in advanced rechargeable batteries. Batter. Supercaps 2(1), 9-36 (2019). https://doi.org/10.1002/batt.201800067

12. K. Yang, S. Zhang, D. Han, M. Xiao, S. Wang, Y. Meng, Multifunctional lithium-sulfur battery separator. Progr. Chem. 30(12), 1942-1959 (2018). https://doi.org/10.7536/ pc180405

13. X.-J. Hong, C.-L. Song, Y. Yang, H.-C. Tan, G.-H. Li, Y.-P. Cai, H. Wang, Cerium based metal-organic frameworks as an efficient separator coating catalyzing the conversion of polysulfides for high performance lithium-sulfur batteries. ACS Nano 13(2), 1923-1931 (2019). https://doi.org/10.1021/acsna no. 8 b08155

14. P. Han, S.-H. Chung, A. Manthiram, Thin-layered molybdenum disulfide nanoparticles as an effective polysulfide mediator in lithium-sulfur batteries. ACS Appl. Mater. Interfaces. 10(27), 23122-23130 (2018). https://doi.org/10.1021/acsam i. $8 \mathrm{~b} 05397$

15. J.S. Lee, W. Kim, J. Jang, A. Manthiram, Sulfur-embedded activated multichannel carbon nanofiber composites for longlife, high-rate lithium-sulfur batteries. Adv. Energy Mater. 7(5), 1601943 (2017). https://doi.org/10.1002/aenm.20160 1943

16. X. Zhang, Y. Zhong, X. Xia, Y. Xia, D. Wang et al., Metalembedded porous graphitic carbon fibers fabricated from bamboo sticks as a novel cathode for lithium-sulfur batteries. ACS Appl. Mater. Interfaces. 10(16), 13598 (2018). https:// doi.org/10.1021/acsami.8b02504

17. J. Liang, C. Yuan, H. Li, K. Fan, Z. Wei, H. Sun, J. Ma, Growth of $\mathrm{SnO}_{2}$ nanoflowers on $n$-doped carbon nanofibers as anode for Li- and Na-ion batteries. Nano-Micro Lett. 10(2), 21 (2018). https://doi.org/10.1007/s40820-017-0172-2

18. L. Hencz, H. Chen, H.Y. Ling, Y. Wang, C. Lai, H. Zhao, S. Zhang, Housing sulfur in polymer composite frameworks for Li-S batteries. Nano-Micro Lett. 11(1), 17 (2019). https://doi. org/10.1007/s40820-019-0249-1

19. T. Gong, R. Qi, X. Liu, H. Li, Y. Zhang, N, F-codoped microporous carbon nanofibers as efficient metal-free electrocatalysts for ORR. Nano-Micro Lett. 11(1), 9 (2019). https ://doi.org/10.1007/s40820-019-0240-x

20. Z. Sun, J. Zhang, L. Yin, G. Hu, R. Fang, H.M. Cheng, L. Feng, Conductive porous vanadium nitride/graphene composite as chemical anchor of polysulfides for lithium-sulfur batteries. Nat. Commun. 8, 14627 (2017). https://doi. org/10.1038/ncomms 14627
21. X. Yao, N. Huang, F. Han, Q. Zhang, H. Wan, J.P. Mwizerwa, C. Wang, X. Xu, High-performance all-solid-state lithiumsulfur batteries enabled by amorphous sulfur-coated reduced graphene oxide cathodes. Adv. Energy Mater. 7(17), 1602923 (2017). https://doi.org/10.1002/aenm.201602923

22. J. Wang, H. Yang, Z. Chen, L. Zhang, J. Liu et al., Doubleshelled phosphorus and nitrogen codoped carbon nanospheres as efficient polysulfide mediator for high-performance lithium-sulfur batteries. Adv. Sci. 5(11), 1800621 (2018). https ://doi.org/10.1002/advs.201800621

23. Q. Zhang, F. Li, J.-Q. Huang, H. Li, Lithium-sulfur batteries: co-existence of challenges and opportunities. Adv. Funct. Mater. 28(38), 1804589 (2018). https://doi.org/10.1002/ adfm.201804589

24. L. Lu, J.T.M. De Hosson, Y. Pei, Three-dimensional micronporous graphene foams for lightweight current collectors of lithium-sulfur batteries. Carbon 144, 713-723 (2019). https ://doi.org/10.1016/j.carbon.2018.12.103

25. C. Tang, Q. Zhang, M.-Q. Zhao, J.-Q. Huang, X.-B. Cheng, G.-L. Tian, H.-J. Peng, F. Wei, Nitrogen-doped aligned carbon nanotube/graphene sandwiches: facile catalytic growth on bifunctional natural catalysts and their applications as scaffolds for high-rate lithium-sulfur batteries. Adv. Mater. 26(35), 6100-6105 (2015). https://doi.org/10.1002/adma.201401243

26. Y. Zhong, X. Xia, S. Deng, D. Xie, S. Shen et al., Spore carbon from aspergillus oryzae for advanced electrochemical energy storage. Adv. Mater. 30, 1805165 (2018). https://doi. org/10.1002/adma.201805165

27. X. Liu, J.-Q. Huang, Q. Zhang, L. Mai, Nanostructured metal oxides and sulfides for lithium-sulfur batteries. Adv. Mater. 29(20), 1601759 (2017). https://doi.org/10.1002/adma.20160 1759

28. J. He, L. Luo, Y. Chen, A. Manthiram, Yolk-shelled C@ $\mathrm{Fe}_{3} \mathrm{O}_{4}$ nanoboxes as efficient sulfur hosts for high-performance lithium-sulfur batteries. Adv. Mater. 29(34), 1702707 (2017). https://doi.org/10.1002/adma.201702707

29. T. Chen, L. Ma, B. Cheng, R. Chen, Y. Hu et al., Metallic and polar $\mathrm{Co}_{9} \mathrm{~S}_{8}$ inlaid carbon hollow nanopolyhedra as efficient polysulfide mediator for lithium-sulfur batteries. Nano Energy 38, 239-248 (2017). https://doi.org/10.1016/j.nanoe n.2017.05.064

30. X. Chen, H.-J. Peng, R. Zhang, T.-Z. Hou, J.-Q. Huang, B. Li, Q. Zhang, An analogous periodic law for strong anchoring of polysulfides on polar hosts in lithium sulfur batteries: S- or Li-binding on first-row transition-metal sulfides? ACS Energy Lett. 2(4), 795-801 (2017). https://doi.org/10.1021/acsenergyl ett. $7 b 00164$

31. J. Xu, T. Lawson, H. Fan, D. Su, G. Wang, Updated metal compounds (MOFs, $-\mathrm{S},-\mathrm{OH},-\mathrm{N},-\mathrm{C}$ ) used as cathode materials for lithium-sulfur batteries. Adv. Energy Mater. 8(10), 1702607 (2018). https://doi.org/10.1002/aenm.201702607

32. L. Zhen, J. Zhang, B. Guan, W. Da, L.M. Liu, W.L. Xiong, A sulfur host based on titanium monoxide@carbon hollow spheres for advanced lithium-sulfur batteries. Nat. Commun. 7, 13065 (2016). https://doi.org/10.1038/ncomms 13065 
33. W. Wu, Z. Ruan, J. Li, Y. Li, Y. Jiang, X. Xu, D. Li, Y. Yuan, K. Lin, In situ preparation and analysis of bimetal Co-doped mesoporous graphitic carbon nitride with enhanced photocatalytic activity. Nano-Micro Lett. 11(1), 10 (2019). https://doi. org/10.1007/s40820-018-0236-y

34. D. Wang, Z. Li, J. Zhou, H. Fang, X. He et al., Simultaneous detection and removal of formaldehyde at room temperature: JanusAu@ZnO@ZIF-8 nanoparticles. Nano-Micro Lett. 10(1), 4 (2018). https://doi.org/10.1007/s40820-017-0158-0

35. S.-Z. Zeng, Y. Yao, X. Zeng, Q. He, X. Zheng, S. Chen, W. $\mathrm{Tu}, \mathrm{J}$. Zou, A composite of hollow carbon nanospheres and sulfur-rich polymers for lithium-sulfur batteries. J. Power Sources 357, 11-18 (2017). https://doi.org/10.1016/j.jpows our.2017.04.092

36. H.H. Nersisyan, S.H. Joo, B.U. Yoo, D.Y. Kim, T.H. Lee et al., Combustion-mediated synthesis of hollow carbon nanospheres for high-performance cathode material in lithium-sulfur battery. Carbon 103, 255-262 (2016). https://doi.org/10.1016/j. carbon.2016.03.022

37. Y. Qu, Z. Zhang, X. Wang, Y. Lai, Y. Liu, L. Jie, A simple SDS-assisted self-assembly method for the synthesis of hollow carbon nanospheres to encapsulate sulfur for advanced lithium-sulfur batteries. J. Mater. Chem. A 1(45), 14306-14310 (2013). https://doi.org/10.1039/C3TA13306K

38. X.H. Xia, Z.Y. Zeng, X.L. Li, Y.Q. Zhang, J.P. Tu, N.C. Fan, H. Zhang, H.J. Fan, Fabrication of metal oxide nanobranches on atomic-layer-deposited $\mathrm{TiO}_{2}$ nanotube arrays and their application in energy storage. Nanoscale 5(13), 6040-6047 (2013). https://doi.org/10.1039/C3nr01606d

39. G. He, S. Evers, X. Liang, M. Cuisinier, A. Garsuch, L.F. Nazar, Tailoring porosity in carbon nanospheres for lithium-sulfur battery cathodes. ACS Nano 7(12), 10920 10930 (2013). https://doi.org/10.1021/nn404439r

40. H. Hu, H. Cheng, Z. Liu, G. Li, Q. Zhu, Y. Yu, In situ polymerized PAN-assisted S/C nanosphere with enhanced highpower performance as cathode for lithium/sulfur batteries. Nano Lett. 15(8), 5116-5123 (2015). https://doi.org/10.1021/ acs.nanolett.5b01294

41. X. Xia, S. Deng, S. Feng, J. Wu, J. Tu, Hierarchical porous $\mathrm{Ti}_{2} \mathrm{Nb}_{10} \mathrm{O}_{29}$ nanospheres as superior anode materials for lithium ion storage. J. Mater. Chem. A 5, 21134-21139 (2017). https://doi.org/10.1039/C7TA07229E

42. X. Xia, S. Deng, D. Xie, Y. Wang, S. Feng, J. Wu, J. Tu, Boosting sodium ion storage by anchoring $\mathrm{MoO}_{2}$ on vertical graphene arrays. J. Mater. Chem. A 6(32), 15546-15552 (2018). https://doi.org/10.1039/C8TA06232C

43. J. Zhan, S. Deng, Y. Zhong, Y. Wang, X. Wang, Y. Yu, X. Xia, $\mathrm{J}$. Tu, Exploring hydrogen molybdenum bronze for sodium ion storage: performance enhancement by vertical graphene core and conductive polymer shell. Nano Energy 44, 265-271 (2018). https://doi.org/10.1016/j.nanoen.2017.12.012

44. M. Ling, W. Yan, A. Kawase, H. Zhao, Y. Fu, V.S. Battaglia, G. Liu, Electrostatic polysulfides confinement to inhibit redox shuttle process in the lithium sulfur batteries. ACS Appl. Mater. Interfaces. 9(37), 31741-31745 (2017). https:// doi.org/10.1021/acsami.7b06485

45. L. Xiaoman, Z. Qinglin, G. Weimin, L. Qinghua, The catalytic activity of manganese dioxide supported on graphene promoting the electrochemical performance of lithium-sulfur batteries. J. Electroanal. Chem. 840, 144-152 (2019). https:// doi.org/10.1016/j.jelechem.2019.03.041 